\title{
Precision Nanomedicine Using Dual PET and MR Temperature Imaging-Guided Photothermal Therapy
}

\author{
Min Zhou ${ }^{1-3}$, Marites Melancon ${ }^{4}$, R. Jason Stafford ${ }^{5}$, Junjie Li ${ }^{1}$, Alpa M. Nick ${ }^{6}$, Mei Tian², Anil K. Sood ${ }^{6,7}$, and Chun $\mathrm{Li}^{1}$ \\ ${ }^{I}$ Department of Cancer Systems Imaging, The University of Texas MD Anderson Cancer Center, Houston, Texas; ${ }^{2}$ Department of \\ Nuclear Medicine and PET Center, The Second Affiliated Hospital, Zhejiang University School of Medicine, Hangzhou, Zhejiang, \\ China; ${ }^{3}$ Institute of Translational Medicine, School of Medicine, Zhejiang University, Hangzhou, China; ${ }^{4}$ Department of \\ Interventional Radiology, The University of Texas MD Anderson Cancer Center, Houston, Texas; ${ }^{5}$ Department of Imaging Physics, \\ The University of Texas MD Anderson Cancer Center, Houston, Texas; ${ }^{6}$ Department of Gynecologic Oncology and Reproductive \\ Medicine, The University of Texas MD Anderson Cancer Center, Houston, Texas; and ${ }^{7}$ Center for RNAi and Non-Coding RNA, \\ The University of Texas MD Anderson Cancer Center, Houston, Texas
}

Imaging-based techniques have enabled the direct integration of noninvasive imaging with minimally invasive interventions such as photothermal therapy (PTT) to improve the precision of treatment. Methods: We investigated the feasibility of PTT for ovarian cancer under the guidance of PET and MR temperature imaging using copper sulfide nanoparticles (CuS NPs). The tumor distribution of the CuS NPs after systemic administration was assessed using highly sensitive, quantifiable PET imaging. Two wavelengths of near-infrared (NIR) lasers-808 and $980 \mathrm{~nm}$-were tested for PTT using noninvasive MR temperature imaging real-time monitoring. Results: The in vivo studies revealed that the 980-nm NIR laser had better photothermal effects than the 808-nm NIR laser. These results were in accord with the histologic findings. In vivo PTT using CuS NPs combined with 980-nm laser irradiation achieved significant tumor ablation compared with no treatment control in both subcutaneous $(P=0.007)$ and orthotopic $(P<0.001)$ models of ovarian cancer with regard to the percentage of necrotic damage. Conclusion: Our results indicate that real-time monitoring of the accuracy of PTT is a promising approach for future clinical translation of this emerging thermal ablation technique.

Key Words: PET; MRI; ovarian cancer; photothermal therapy; CuS nanoparticles

J Nucl Med 2016; 57:1778-1783

DOI: 10.2967/jnumed.116.172775

$\mathbf{P}$ recision medicine is an emerging approach for cancer treatment and prevention that takes into account individual variability in genes, environment, and lifestyle $(1,2)$. Image-guided therapy makes cancer treatment less invasive and more precise, which can lead to the selective targeting of cancer cells and the minimization of damage to normal tissues $(3,4)$. One disease in which imageguided therapy has the potential to improve outcomes is ovarian cancer $(\mathrm{OvC})$. OvC is one of the most lethal gynecologic malignancies, with 21,290 new cases and 14,180 deaths estimated in

Received Feb. 1, 2016; revision accepted Apr. 26, 2016.

For correspondence or reprints contact: Chun Li, Department of Cancer Systems Imaging, Unit 1907, 1881 East Rd., 3SCR4.3636, Houston, TX 77054-1907.

E-mail: cli@mdanderson.org

Published online Jun. 9, 2016.

COPYRIGHT (C 2016 by the Society of Nuclear Medicine and Molecular Imaging, Inc. the United States for 2015 (5). The high rate of lethality from OvC is primarily due to the advanced stage of disease at diagnosis. Earlystage OvC can be cured with current therapies in up to $90 \%$ of patients (6), but the cure rate drops substantially in patients with advanced disease. Only about $30 \%$ of patients with advanced-stage $\mathrm{OvC}$ survive $5 \mathrm{y}$ after the initial diagnosis (5). Unlike other cancers, OvC can spread by direct invasion to adjacent organs or by transport throughout the peritoneal cavity via peritoneal fluid $(7,8)$. Because of the diffuse nature of $\mathrm{OvC}$, surgery alone or combined with chemotherapy rarely leads to cure. Although $70 \%$ of patients with $\mathrm{OvC}$ initially experience a response to therapy, most of these patients eventually die of disease recurrence (7). Du Bois et al. (9) showed that complete resection had significantly improved overall survival compared with patients with optimal debulking (small residual tumor burden of 1-10 $\mathrm{mm}$ ) and that the impact of optimal debulking showed a smaller but significant prognostic effect compared with suboptimal debulking (macroscopic residual disease exceeding $1 \mathrm{~cm}$ ) in patients with advanced OvC. These results suggest that image-guided intervention techniques that offer accurate debulking would be beneficial in OvC.

Near-infrared (NIR) laser-induced photothermal therapy (PTT), which has few adverse effects and provides a minimally invasive treatment for inoperable tumors, has undergone rapid development because of the minor attenuation and favorable biosafety of NIR in tissues (10). Nanomaterials with strong NIR absorption can convert light energy into thermal energy at the tumor site to safely ablate tumor cells. Copper sulfide nanoparticles (CuS NPs) are promising photothermal agents because of their high tumor uptake, low cost, and high photothermal conversion efficiency $(11,12)$. Unlike gold nanostructures, this type of absorption is not affected by the surrounding environment (13). Successful PTT should have the following features: targeted delivery of photothermal-conducting NPs to the tumor for effective thermal damage with NIR laser exposure, while keeping the surrounding normal tissues healthy; accurate thermal dose calculation; and noninvasive real-time monitoring of heat profile and response to therapy in a given target volume $(10,14)$.

MR temperature imaging (MRTI) allows real-time assessment of thermal-based therapies throughout the body $(15,16)$. This assessment is critical for ensuring adequate thermal dose delivered to the tumor while avoiding damage to normal tissue by overheating. In addition to MRTI, PET imaging could also be used to improve the precision of PTT. PET is a quantifiable imaging modality that is 
broadly used in the clinic because of its high sensitivity, wide range of available radionuclides, and unlimited depth penetration (17). Quantitative biodistribution data enable the optimization of cancer treatment, for example, adjustment of the drug concentration and avoidance of damage to surrounding healthy tissue (18).

In this study, we first used PET imaging to assess the tumor distribution of the CuS NPs after intravenous injection. Then, we used MRTI to monitor PTT of OvC in vivo. We compared the tumor temperature changes and therapeutic effects of $980-\mathrm{nm}$ versus $808-\mathrm{nm}$ NIR laser irradiation in CuS NP-mediated PTT of OvC in vivo using MRTI. Our data showed that CuS NPs plus 980-nm laser irradiation effectively destroyed tumor cells in both subcutaneous and orthotopic OvC models. These studies indicate that PET and MRTI dual imageguided PTT using CuS NPs combined with a 980-nm NIR laser is a promising therapeutic approach in OvC.

\section{MATERIALS AND METHODS}

\section{Synthesis and Characterization of CuS NPs and ${ }^{64} \mathrm{Cu}-\mathrm{CuS}$ NPs}

The preparation and characterization of $\mathrm{CuS}$ NPs and ${ }^{64} \mathrm{Cu}$-labeled $\mathrm{CuS}$ NPs $\left({ }^{64} \mathrm{Cu}-\mathrm{CuS} \mathrm{NPs}\right)$ were performed according to previously reported procedures (19). Hollow gold nanospheres (HAuNS) reported in the current studies were coated with polyethylene glycol as described before (20). The average diameter of the NPs was $12.4 \mathrm{~nm}$, and their hydrodynamic diameter was $30.1 \mathrm{~nm}$ (Supplemental Figs. 1A and 1B; supplemental materials are available at http://jnm.snmjournals.org). The CuS NPs had a strong NIR absorption band centered at $980 \mathrm{~nm}$, with an extinction value of 1.89 optical density (OD) at $100 \mu \mathrm{g} / \mathrm{mL}(\sim 25 \mathrm{nM}$ CuS NPs). The value at $808 \mathrm{~nm}$ was 1.24 OD (Supplemental Fig. 1C).

\section{Animal Care and Tumor Models}

All animal procedures were approved by The University of Texas MD Anderson Cancer Center Institutional Animal Care and Use Committee. Mice (female nude, 8-12 wk) were supplied by Charles River. To establish a subcutaneous nude mouse model of OvC, HeyA8 cells $\left(5 \times 10^{5}\right.$ cells/mouse) were injected subcutaneously into the thighs of the mice. Seven to $10 \mathrm{~d}$ later, the tumor size reached $5-8 \mathrm{~mm}$ in diameter. To obtain an orthotopic OvC model, Skov3-ip1 cells $\left(5 \times 10^{5}\right.$ cells/mouse) were injected into the peritoneal cavity. The tumors were monitored by an IVIS 200 imaging instrument (Xenogen Corp., USA) to measure the tumor burden. Tumor size reached $1-3 \mathrm{~mm}$ in diameter in $12-15 \mathrm{~d}$ after inoculation.

\section{PET Imaging and Biodistribution of ${ }^{64} \mathrm{Cu}-\mathrm{CuS}$ NPs in HeyA8 Tumor Model}

Female nude mice bearing subcutaneous HeyA8 tumors received tail vein injection of ${ }^{64} \mathrm{Cu}-\mathrm{CuS}$ NPs $(0.74 \mathrm{MBq} /$ mouse in $200 \mu \mathrm{L})$. The animals were anesthetized with $2 \%$ isoflurane and placed prone, and small-animal PET/CT images were acquired at 1, 6, and $24 \mathrm{~h}$ after injection of the radiolabeled nanoparticles using an Inveon micro-PET/CT scanner (Siemens Preclinical Solution). The small-animal PET and CT images were generated separately and then fused using Inveon Research Workplace. For data analysis, the region of interest was manually drawn covering the whole tumor on the CT images and copied to the corresponding PET images. Twenty-four hours after NP injection, the mice were killed and major organs were removed, weighed, and counted for radioactivity (Packard Cobra, USA). Organ uptake of radiotracer was expressed as a percentage injected dose per gram of tissue (\%ID/g).

\section{MRTI-Guided PTT of Subcutaneous OvC Tumors}

Mice with HeyA8 subcutaneous tumors on both thighs were treated when the tumors reached 5-8 $\mathrm{mm}$ in diameter. Mice ( $n=4 /$ group $)$ were injected intravenously with CuS NPs (400 $\mu \mathrm{g} / \mathrm{mL}, 200 \mu \mathrm{L} /$ mouse), and
NIR laser treatment was delivered $24 \mathrm{~h}$ later $\left(2 \mathrm{~W} / \mathrm{cm}^{2}, 2 \mathrm{~min}\right)$. Tumors on the left and right thighs of the mice were irradiated with 808- and 980-nm lasers, respectively. During the laser irradiation, a 1.5-T clinical MRI scanner (GE Healthcare) equipped with temperature monitoring and a thermal mapping system (Excite HD, USA) was used to locate the $\mathrm{CuS}$ NPs and monitor the temperature change in the tumor area. A multiple, fast-gradient, refocused echo was used, with 16 echoes at echo times ranging from 2 to $60 \mathrm{~ms}$ for each repetition time. $\mathrm{T}_{2}{ }^{*}$ maps were calculated using the Steiglitz-McBride algorithm, which can provide accurate and precise $\mathrm{T}_{2}{ }^{*}$ estimates. This technique also calculates the proton resonant frequency to estimate temperature changes, thereby providing simultaneous $\mathrm{T}_{2} *$ mapping and MRTI. Twenty-four hours after treatment, the tumors were removed and processed for hematoxylin and eosin staining. The temperature change of the tumors was monitored by an infrared thermal imaging camera (FLIR i7; FLIR Systems Inc.) during laser treatment.

\section{In Vivo PTT of Orthotopic OvC Tumors}

Skov3-ip1 tumor-bearing mice (orthotopic model) were treated when the tumor reached $1-3 \mathrm{~mm}$ in diameter. The tumor-bearing mice were randomly allocated to 3 groups ( $n=4$ mice/group). Mice in the PTT group (group 1) and laser-only group (group 2) were injected intravenously with CuS NPs (400 $\mu \mathrm{g} / \mathrm{mL}, 200 \mu \mathrm{L} /$ mouse) and saline, respectively. Mice in the control group (group 3) were injected with saline intravenously. NIR laser treatment $\left(980 \mathrm{~nm}, 2 \mathrm{~W} / \mathrm{cm}^{2}, 2 \mathrm{~min}\right)$ was delivered $24 \mathrm{~h}$ after injection (groups 1 and 2). Twenty-four hours after laser treatment, the mice were killed and the tumors and surrounding intestine sectioned for hematoxylin and eosin staining and histologic examination.

\section{Evaluation of Toxicity}

Toxicity experiments were performed with 8-wk-old male Swiss mice (20-25 g). Mice $(n=3)$ were injected intravenously with $\mathrm{CuS}$ NPs (400 $\mu \mathrm{g} / \mathrm{mL}, 8 \mathrm{OD}, 200 \mu \mathrm{L} /$ mouse). The mice were killed by $\mathrm{CO}_{2}$ overexposure, and necropsy was performed $14 \mathrm{~d}$ later. Representative organs, including the liver, spleen, and kidneys, were stained with hematoxylin and eosin and images were analyzed for potential adverse effects.

\section{Statistical Analysis}

Differences in tumor necrosis percentages between different study conditions and mouse groups were analyzed using the 2-tailed Student $t$ test. Differences between groups were considered statistically significant at a $P$ value of less than 0.05 .

\section{RESULTS}

\section{Comparison of Nanomaterials for Photothermal Effect}

Figure 1A compares the optical extinction spectra of CuS NPs, HAuNS, and single-wall carbon nanotubes (SWCNTs) at the same concentration of $100 \mu \mathrm{g} / \mathrm{mL}$. At $980 \mathrm{~nm}, \mathrm{CuS}$ NPs displayed an optical extinction value $(\mathrm{OD}=1.89)$ more than twice that of HAuNS (OD $=0.95)$ and approximately 6 times that of SWCNTs $(\mathrm{OD}=0.33)$. Then, we compared the temperature changes in aqueous solutions of these nanomaterials under $980-\mathrm{nm}$ continuous wavelength laser irradiation. Because of the high NIR absorbance at $980 \mathrm{~nm}$, exposure of an aqueous solution of CuS NPs $(100 \mu \mathrm{g} / \mathrm{mL})$ to the NIR laser light $\left(2 \mathrm{~W} / \mathrm{cm}^{2}\right)$ for $4 \mathrm{~min}$ rapidly elevated the temperature of the solution from $22.1^{\circ} \mathrm{C}$ to $99.85^{\circ} \mathrm{C}$ (an increase of $77.54^{\circ} \mathrm{C}$ ), as shown in Figure 1B. In contrast, under the same conditions, increases in temperature to only $62.85^{\circ} \mathrm{C}$ and $47.09^{\circ} \mathrm{C}$ after $10 \mathrm{~min}$ of NIR light irradiation were observed with HAuNS and SWCNTs, respectively. These data show that CuS NPs are an ideal photothermal converter using a 980-nm laser. 


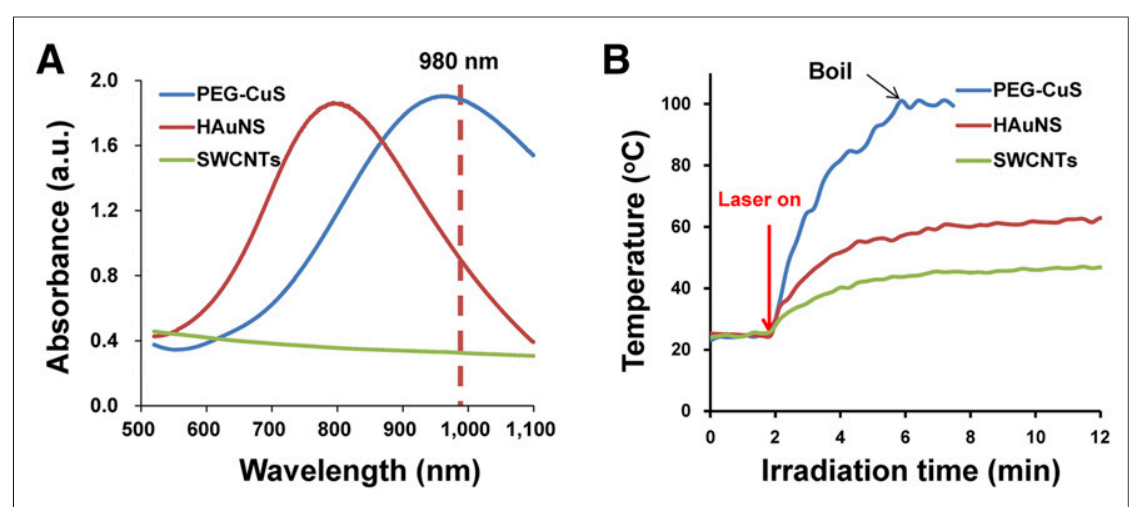

FIGURE 1. Optical absorbance and NIR laser-induced temperature change of different photothermal coupling nanomaterials. (A) Optical absorbance of CuS NPs, HAuNS, and SWCNTs at same concentration $(100 \mu \mathrm{g} / \mathrm{mL})$. (B) Corresponding temperature change curve of different nanomaterials under 980-nm continuous NIR laser irradiation (laser power density, 2 W/cm²).

$6 \mathrm{~mm}$ ) in tumors beneath the skin during the 980-nm (Figs. 3A and 3C) and 808-nm (Figs. 3B and 3D) laser treatments. Maximal temperature elevations under the 980-nm NIR laser were found to occur $1 \mathrm{~mm}$ under the skin surface. During the first $40 \mathrm{~s}$ of heating, the temperature increased rapidly because of the intense localized heating before the thermal energy dissipated into the surrounding tissues. Then, the temperature elevated gradually from 40 to $120 \mathrm{~s}$. Lower temperatures were observed when the distance between the skin and the targeted area increased. This result indicated that a higher thermal dosage with a short irradiation time may provide optimal heating of the tumor and minimize the surrounding healthy tissue damage. Much lower temperatures were found in the tumor when the 808-nm NIR

\section{Optimization of Laser for Photothermal Effect In Vitro}

Next, we compared in vitro cell killing induced by the $\mathrm{CuS} \mathrm{NP}-$ mediated photothermal effect using lasers with 808- and 980-nm wavelengths. No obvious difference was found between untreated cells (no laser, no CuS NPs) and cells treated with CuS NPs alone (Supplemental Fig. 2) at $24 \mathrm{~h}$ after treatment. Only a small portion of cells were killed by the 980 - and 808 -nm lasers alone or the $\mathrm{CuS}$ NPs plus the 808-nm laser. However, cells treated with CuS NPs and the $980-\mathrm{nm}$ laser had substantially reduced cell viability. Thus, the 980-nm laser has a better therapeutic effect on cancer cells than the 808-nm laser.

\section{Quantitative Analysis of Tumor Uptake of CuS NPs: In Vivo PET Imaging and Organ Biodistribution After Intravenous Injection}

Small-animal PET images (Fig. 2; Supplemental Fig. 3) showed a strong signal in the liver at all time points, indicating high accumulation of the ${ }^{64} \mathrm{Cu}-\mathrm{CuS}$ NPs in the organs of the reticuloendothelial system. Signals in the tumor increased over time, with the highest activity found at $24 \mathrm{~h}$ after injection. Quantitative analysis of the small-animal PET/CT imaging data showed tumor uptake values of 2.1, 4.5, and $6.4 \% \mathrm{ID} / \mathrm{g}$ at 1,6 , and $24 \mathrm{~h}$ after injection of ${ }^{64} \mathrm{Cu}-\mathrm{CuS}$ NPs, respectively. Biodistribution data (Supplemental Fig. 4) further confirmed the imaging finding, which showed a tumor uptake value of $5.99 \pm 1.34 \% \mathrm{ID} / \mathrm{g}$ at $24 \mathrm{~h}$ after injection. Taken together, the high tumor accumulation of ${ }^{64} \mathrm{Cu}-\mathrm{CuS}$ NPs indicates the feasibility of PTT using CuS NPs in OvC at $24 \mathrm{~h}$ after injection.

\section{MRTI-Guided Photothermal Ablation of Subcutaneous OvC Tumors In Vivo}

Supplemental Figure 5A shows the scheme of the in vivo laser treatment of the tumor-bearing mice. Supplemental Figure 5B shows a representative T2* MR image of the tumors. As shown in the representative MR temperature images of tumors in Supplemental Figure 5C, the temperatures of the tumors increased during the laser treatment, reaching a peak at approximately $120 \mathrm{~s}$ and then decreasing after the laser was turned off. The laser penetration depth of the 980-nm laser was $6.0 \mathrm{~mm}$, which was 3 times longer than that of the $808-\mathrm{nm}$ laser $(2.0 \mathrm{~mm})$. These data show that the $980-\mathrm{nm}$ laser is a better light source than the 808-nm laser for coupling with CuS NP-mediated PTT.

Figure 3 shows MR temperature images and plots of temperature variation versus laser irradiation time at different depths (1, 2, 4, and laser was used. We also used a thermal camera to measure the tumor temperatures during laser irradiation (Supplemental Fig. 6). Only skin temperature can be captured with the thermal camera, and these results were similar to the temperature data obtained using MRTI. The tumors reached a maximum temperature of $56.1^{\circ} \mathrm{C}$ and $36.7^{\circ} \mathrm{C}$ with the 980-nm laser and the 808-nm laser, respectively.

In the case of the 980-nm laser-treated subcutaneous HeyA8 OvC, tumor cell necrosis was present in most areas of the examined tumor slide. Moreover, damage to the cancer cells, such as cell shrinkage, eosinophilic cytoplasm, and nuclear damage, was also found (Fig. 4A). However, for the 808-nm laser-treated HeyA8 tumors, most of the tumor cells were still alive and there were no noticeable differences in the tumor cells' size and shape, nuclear modifications, or necrosis after irradiation (Fig. 4B). The percentage of necrosis in tumors treated with the 980-nm laser was significantly greater than that in tumors treated with the 808-nm laser $(P=0.003)$ (Fig. 4C). These results showed that although treatment with an 808 -nm laser leads to a temperature increase that causes partial tumor damage, 980-nm laser treatment can kill many more cancer cells because of its greater temperature increase and deeper penetration.

\section{In Vivo Photothermal Ablation of Orthotopic OvC Tumors}

Because our subcutaneous OvC model data showed that the tumors treated with a 980-nm laser had significantly larger areas of

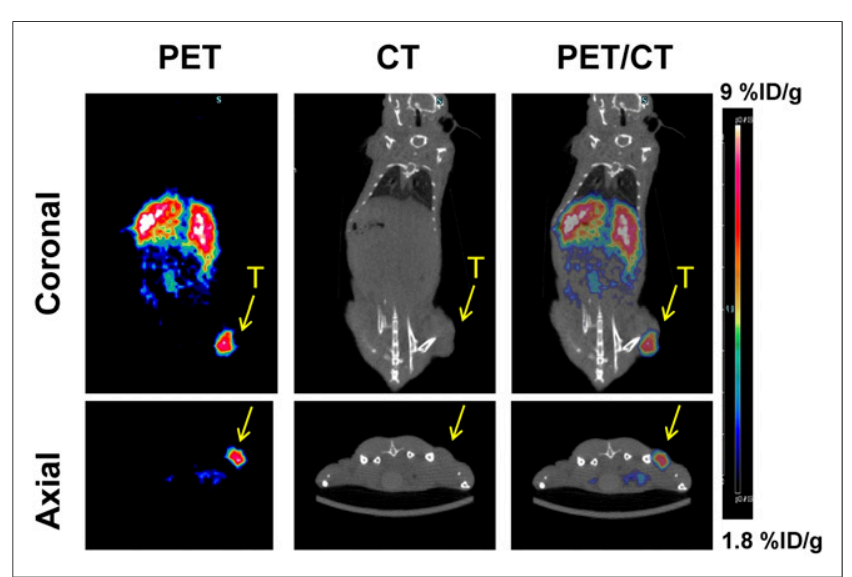

FIGURE 2. PET/CT images acquired at $24 \mathrm{~h}$ after intravenous injection of ${ }^{64} \mathrm{Cu}-\mathrm{CuS}$ NPs in mice bearing subcutaneous HeyA8 OvC tumors (T). 

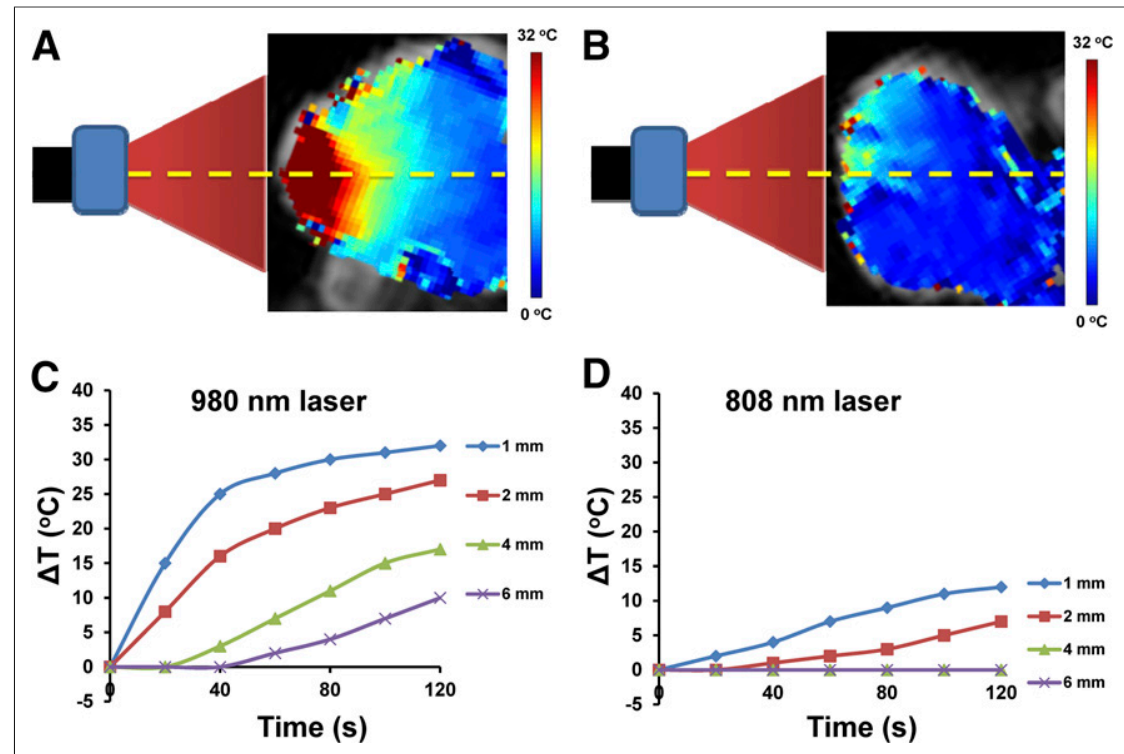

FIGURE 3. MRTI-guided real-time tumor temperature elevation with laser irradiation in subcutaneous HeyA8 ovarian tumor-bearing mouse model. Measured temperature rise plotted as function of depth. Measured temperature rise in region of interest at different depths $(1,2,4$, and $6 \mathrm{~mm}$ ) beneath tumor skin for 980-nm (A and C) and 808-nm (B and D) laser treatment.

PET provides the possibility of not only assessing quantitatively the accumulation of nanoparticles in the tumor to ensure adequate uptake for PTT but also guiding the application of PTT. Given that combined PET/MRI systems with integrated software and hardware are now clinically available, an MRI-compatible PTT technique may have advantages over other currently used thermal ablation techniques such as radiofrequency ablation in monitoring the progress of treatment.

NP-mediated thermal conversion reaches its highest temperature when the absorbance wavelength is equal to the illumination laser wavelength, which means that the stronger the absorbance at the illumination laser wavelength, the higher the temperature of the photothermal materials. HAuNS and SWCNTs have also been proposed as photothermal agents for in vivo PTT (2024). However, these agents have low absorption at $980 \mathrm{~nm}$. In our experiments, the optic absorption value of CuS NPs was almost twice and 6-fold that of HAuNS and

necrosis than those treated with an 808-nm laser, we chose to use the 980-nm laser to examine the therapeutic effect of CuS NP-mediated PTT in an orthotopic Skov3-ip1 OvC model (Fig. 5). The tumor treated with the 980-nm laser plus CuS NPs demonstrated prominent necrosis. We also found a large amount of cell shrinkage, cytoplasm loss, cell death, and serious tumor tissue structure damage in the treated tumor samples (Fig. 5C). These results demonstrated a successful photothermal killing effect on the cancer cells. The mice that were untreated or treated with laser alone had no obvious changes in their tumor tissues (Figs. 5A and 5B), indicating that the 980-nm laser irradiation alone, without CuS NPs, cannot kill tumor cells. The percentage of necrosis in tumors treated with $\mathrm{CuS}$ NPs plus the 980-nm laser was significantly greater than that in tumors treated with NIR laser only $(P=0.00017)$ and in the untreated (control) tumors $(P<0.0001)$ (Fig. 5D). We also investigated alterations in the normal intestine surrounding the tumors after treatment (Supplemental Figs. 7A-7C). No obvious changes were found at $24 \mathrm{~h}$ after treatment. Taken together, these findings demonstrate that $\mathrm{CuS}$ NP plus 980-nm laser treatment has potent antitumor effects against OvC tumors but does not harm nearby healthy tissues.

We assessed the acute toxicity of CuS NPs in the treated mice with orthotopic OvC. No obvious abnormal behavior was seen in the CuS NP-injected mice during the experiments. As shown in Supplemental Figure 8, no obvious hydropic damage or lesions were observed in hematoxylin and eosin-stained slices at $14 \mathrm{~d}$ after injection of CuS NPs. Overall, there were no evident histopathologic abnormalities in the CuS NP-treated mice at the test dosage (3.2 mg/kg, 8 OD, $200 \mu \mathrm{L} /$ mouse).

\section{DISCUSSION}

Our results indicate that CuS NPs in combination with 980-nm NIR laser-mediated PTT is a novel and effective therapeutic approach for treating $\mathrm{OvC}$. This technique is further enhanced by the use of PET for precise localization of the nanoparticles and of MRTI for temperature monitoring.
SWCNTs, respectively, at $980 \mathrm{~nm}$. The corresponding photothermal conversion efficiency of $\mathrm{CuS}$ NPs was also much higher than that of HAuNS and SWCNTs $\left(99.85^{\circ} \mathrm{C}\right.$ vs. $62.85^{\circ} \mathrm{C}$ and $47.09^{\circ} \mathrm{C}$, respectively). ${ }^{64} \mathrm{Cu}-\mathrm{CuS}$ NPs also exhibited high uptake in a HeyA8 subcutaneous tumor model. These results motivated us to investigate the potential of CuS NPs in the in vivo PTT of OvC with a $980-n m$ laser.

Because the photothermal effect is dependent on laser wavelength in PTT, it is vital to optimize the laser wavelength to achieve maximal therapeutic efficacy. We assessed the PTT effects of 2

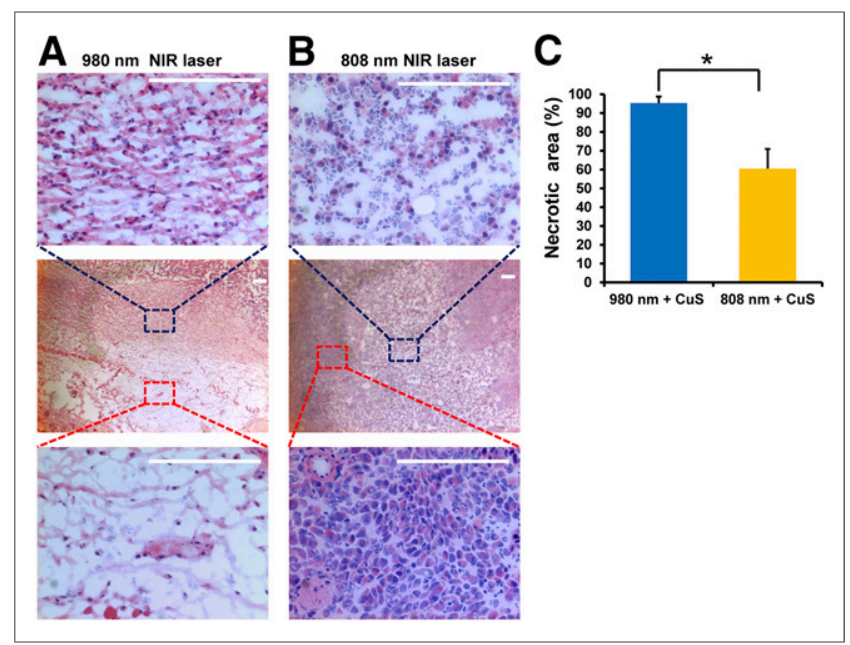

FIGURE 4. In vivo PTT of subcutaneous HeyA8 ovarian tumor-bearing mice intravenously injected with CuS NPs (8 OD). (A and B) Representative hematoxylin and eosin-stained images of tumors removed $24 \mathrm{~h}$ after 980 - and 808-nm NIR laser treatment (2 W/cm² for $2 \mathrm{~min})$. Scale bar $=100 \mu \mathrm{m}$. (C) Quantitative analysis of percentage of necrosis induced by $980-$ or $808-\mathrm{nm}$ laser treatments. Amount of necrosis is presented as percentage of whole tumor area. Error bars represent $\operatorname{SD}(n=3)$. ${ }^{*}$ Statistical significance relative to control $(P<0.01)$. 


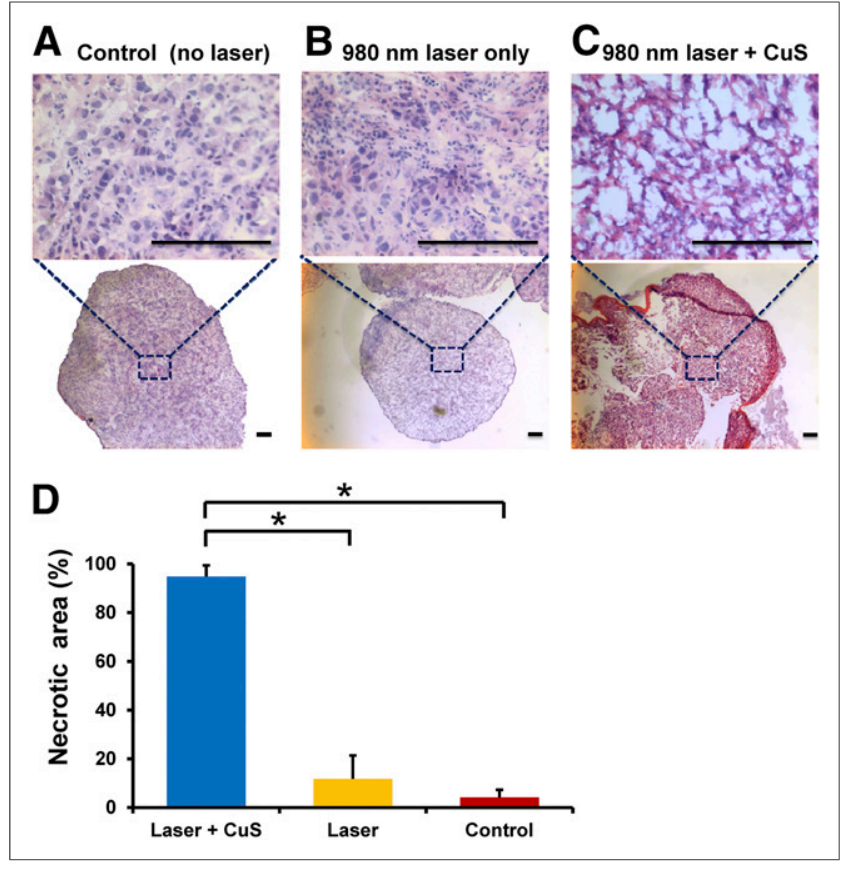

FIGURE 5. CuS NPs induced photothermal destruction of orthotopic Skov3-ip1 ovarian tumors in vivo. Representative hematoxylin and eosin-stained images of tumors (A-C) with/without 980-nm NIR laser irradiation ( $2 \mathrm{~W} / \mathrm{cm}^{2}$ for $2 \mathrm{~min}$ ). Tumors from mice treated with CuS NPs plus $980-\mathrm{nm}$ laser irradiation showed characteristics of significant cellular damage. None of these characteristics was found in surrounding normal cells. Scale bar $=100 \mu \mathrm{m}$. (D) Quantitative analysis of percentage of necrosis induced by CuS NPs plus 980-nm laser treatment, $980-\mathrm{nm}$ laser treatment alone, and no treatment control.

different lasers with different wavelengths, 808 and 980 nm. First, ultraviolet-visible spectra showed that CuS NPs have stronger absorption at $980 \mathrm{~nm}$ than at $808 \mathrm{~nm}$. Second, our in vitro study demonstrated much more tumor cell damage when the 980-nm laser was used. Finally, we chose a subcutaneous HeyA8 tumor model to investigate variations in temperature and penetration depth between 980- and 808-nm laser irradiation. We monitored the local temperature change using a real-time MRTI system during the laser irradiation. In our experiments, we found that the temperatures of the tumors in mice injected with $\mathrm{CuS}$ NPs rapidly elevated to $56.1^{\circ} \mathrm{C}$ under 980 -nm laser exposure, but the temperatures of the tumors treated with $808-\mathrm{nm}$ laser irradiation were much lower. Moreover, the penetration of the 980-nm laser was deeper than that of the 808-nm laser. The histology experiments were consistent with the MRI results, demonstrating more tumor cell necrosis with 980-nm laser treatment. Therefore, realtime monitoring of tumor temperature is critical to predict the therapeutic outcome of PTT. To the best of our knowledge, this is the first study using dual PET/MRTI to guide NP-mediated PTT to achieve effective thermal ablation of tumors.

The 980-nm laser was next used to treat OvC tumors in an orthotopic Skov3-ip1 model. Histologic analysis showed that treatment with $\mathrm{CuS}$ NPs and a 980-nm laser induced large necrotic areas in the mouse tumors. These results indicate that $\mathrm{CuS}$ NPs in combination with 980-nm laser irradiation could mediate effective PTT. Histologic analysis also demonstrated no significant change in the surrounding normal tissues $24 \mathrm{~h}$ after treatment.
Furthermore, we assessed the acute toxicity of the CuS NPs in vivo. No obvious side effects of the CuS NPs were noted in the treated mice during the experiments. Histopathologic analysis of major organs (liver, spleen, and kidney) did not show any significant evidence of histopathologic abnormalities in the $\mathrm{CuS} \mathrm{NP}$-treated mice at the test dosage $(3.2 \mathrm{mg} / \mathrm{kg}, 8 \mathrm{OD}$, $200 \mu \mathrm{L} /$ mouse). Further studies are needed to systematically investigate the acute and chronic toxicity of CuS NPs after systemic administration.

\section{CONCLUSION}

In this study, we present a precision nanomedicine approach based on dual PET/MR temperature image-guided PTT in OvC tumor-bearing mice. Quantification of the data analyzed by PET imaging and biodistribution demonstrated that sufficient $\mathrm{CuS}$ NPs were accumulated in the tumor sites for PTT. With the use of MRTI, real-time temperature change and diffusion of the NP-treated tumors during the laser irradiation can be monitored precisely, and thus damage to the surrounding healthy tissues can be avoided efficiently. Systemic administration of $\mathrm{CuS}$ NPs combined with 980-nm laser treatment demonstrated a highly efficient therapeutic effect on $\mathrm{OvC}$ in both subcutaneous and orthotopic models.

\section{DISCLOSURE}

The costs of publication of this article were defrayed in part by the payment of page charges. Therefore, and solely to indicate this fact, this article is hereby marked "advertisement" in accordance with 18 USC section 1734. This work was supported in part by National Institutes of Health grant U54 CA151668 and by the John S. Dunn Foundation. The Research Animal Support Facility and High Resolution Electron Microscopy Facility are supported by a Cancer Center Support grant from the National Institutes of Health (P30CA016672). No other potential conflict of interest relevant to this article was reported.

\section{ACKNOWLEDGMENTS}

We thank Dawn Chalaire for editing the manuscript.

\section{REFERENCES}

1. Jameson JL, Longo DL. Precision medicine: personalized, problematic, and promising. N Engl J Med. 2015;372:2229-2234.

2. Ashley EA. The precision medicine initiative: a new national effort. JAMA. 2015;313:2119-2120.

3. Penet MF, Krishnamachary B, Chen Z, Jin J, Bhujwalla ZM. Molecular imaging of the tumor microenvironment for precision medicine and theranostics. Adv Cancer Res. 2014;124:235-256.

4. Manning HC. World Molecular Imaging Congress 2015: precision medicine visualized. Mol Imaging Biol. 2015;17:295-296.

5. Siegel RL, Miller KD, Jemal A. Cancer statistics, 2015. CA Cancer J Clin. 2015;65:5-29.

6. Smith RA, Manassaram-Baptiste D, Brooks D, et al. Cancer screening in the United States, 2015: a review of current American cancer society guidelines and current issues in cancer screening. CA Cancer J Clin. 2015; 65:30-54.

7. Naora H, Montell DJ. Ovarian cancer metastasis: integrating insights from disparate model organisms. Nat Rev Cancer. 2005;5:355-366.

8. Kanchi $\mathrm{KL}$, Johnson $\mathrm{KJ}$, Lu C, et al. Integrated analysis of germline and somatic variants in ovarian cancer. Nat Commun. 2014;5:3156. 
9. du Bois A, Reuss A, Pujade-Lauraine E, Harter P, Ray-Coquard I, Pfisterer J. Role of surgical outcome as prognostic factor in advanced epithelial ovarian cancer: a combined exploratory analysis of 3 prospectively randomized phase 3 multicenter trials-by the Arbeitsgemeinschaft Gynaekologische Onkologie Studiengruppe Ovarialkarzinom (AGO-OVAR) and the Groupe d'Investigateurs Nationaux Pour les Etudes des Cancers de l'Ovaire (GINECO). Cancer. 2009;115:1234-1244.

10. Melancon MP, Zhou M, Li C. Cancer theranostics with near-infrared lightactivatable multimodal nanoparticles. Acc Chem Res. 2011;44:947-956.

11. Tian Q, Jiang F, Zou R, et al. Hydrophilic Cu9S5 nanocrystals: a photothermal agent with a $25.7 \%$ heat conversion efficiency for photothermal ablation of cancer cells in vivo. ACS Nano. 2011;5:9761-9771.

12. Zhou M, Ku G, Pageon L, Li C. Theranostic probe for simultaneous in vivo photoacoustic imaging and confined photothermolysis by pulsed laser at 1064 $\mathrm{nm}$ in 4T1 breast cancer model. Nanoscale. 2014;6:15228-15235.

13. Li Y, Lu W, Huang Q, Huang M, Li C, Chen W. Copper sulfide nanoparticles for photothermal ablation of tumor cells. Nanomedicine (Lond). 2010;5:1161-1171.

14. Melancon MP, Lu W, Zhong M, et al. Targeted multifunctional gold-based nanoshells for magnetic resonance-guided laser ablation of head and neck cancer. Biomaterials. 2011;32:7600-7608.

15. Quesson B, de Zwart JA, Moonen CT. Magnetic resonance temperature imaging for guidance of thermotherapy. J Magn Reson Imaging. 2000;12:525-533.

16. Fuentes D, Walker C, Elliott A, Shetty A, Hazle JD, Stafford RJ. Magnetic resonance temperature imaging validation of a bioheat transfer model for laser-induced thermal therapy. Int J Hyperthermia. 2011;27:453-464.
17. Jadvar H. Molecular imaging of prostate cancer with PET. J Nucl Med. 2013; 54:1685-1688.

18. Matthews PM, Rabiner EA, Passchier J, Gunn RN. Positron emission tomography molecular imaging for drug development. Br J Clin Pharmacol. 2012;73:175-186.

19. Zhou M, Zhang R, Huang M, et al. A chelator-free multifunctional $\left[{ }^{64} \mathrm{Cu}\right] \mathrm{CuS}$ nanoparticle platform for simultaneous micro-PET/CT imaging and photothermal ablation therapy. J Am Chem Soc. 2010;132:15351-15358.

20. Lu W, Zhang G, Zhang R, et al. Tumor site-specific silencing of NF-kappaB p65 by targeted hollow gold nanosphere-mediated photothermal transfection. Cancer Res. 2010;70:3177-3188.

21. Lu W, Melancon MP, Xiong CY, et al. Effects of photoacoustic imaging and photothermal ablation therapy mediated by targeted hollow gold nanospheres in an orthotopic mouse xenograft model of glioma. Cancer Res. 2011;71:61166121.

22. Melancon MP, Lu W, Yang Z, et al. In vitro and in vivo targeting of hollow gold nanoshells directed at epidermal growth factor receptor for photothermal ablation therapy. Mol Cancer Ther. 2008;7:1730-1739.

23. Antaris AL, Robinson JT, Yaghi OK, et al. Ultra-low doses of chirality sorted $(6,5)$ carbon nanotubes for simultaneous tumor imaging and photothermal therapy. ACS Nano. 2013;7:3644-3652.

24. Moon HK, Lee SH, Choi HC. In vivo near-infrared mediated tumor destruction by photothermal effect of carbon nanotubes. ACS Nano. 2009;3:37073713 . 